\title{
Estudo de indicadores de saúde ambiental e de saneamento em cidade do Norte do Brasil
}

\section{Study of indicators of environmental health and sanitation in Northern city of Brazil}

\author{
Maria Lúcia Calijuri \\ Engenheira Civil. Professora Titular da Universidade Federal de Viçosa (UFV/MG)
}

\author{
Aníbal da Fonseca Santiago \\ Engenheiro Ambiental. Mestre em Hidráulica e Saneamento pela Escola de Engenharia de São Carlos da Universidade de São Paulo (EESC/USP) \\ Rodrigo de Arruda Camargo \\ Graduando em Engenharia Ambiental da UFV/MG
}

Ronan Fernandes Moreira Neto

Graduando em Engenharia Ambiental pela UFV/MG

\section{Resumo}

A qualidade de vida de uma população está diretamente relacionada com a infraestrutura urbana em que a mesma está inserida. Nesse sentido, o objetivo do estudo foi avaliar a saúde pública da área urbana do município de Tucuruí no Paraná, sob o ponto de vista das condições do saneamento ambiental e a composição de um sistema com menor quantidade de variáveis para discriminar áreas com distintos níveis sanitário-ambientais, e relacionar esses indicadores com a ocorrência de agravos à saúde nessas áreas. O município de Tucuruí foi selecionado para a área de estudo devido à deficiência de sua infraestrutura sanitária, alta taxa de crescimento da população, e a forte incidência de doenças tropicais relacionadas diretamente com as condições sanitárias.

Palavras-chave: indicadores; saúde ambiental; saneamento; norte do Brasil.

\section{Abstract}

The quality of life from a population is directly related to urban infrastructure on which it is embedded. Accordingly, the objective of the study was to evaluate the public health in the municipality of Tucuruí in Paraná, under conditions of environmental sanitation to the composition of a system with a smaller number of variables to discriminate areas with different levels of sanitary and environmental health, and relate these indicators with the occurrence of diseases related to health in these areas. The municipality of Tucuruí was selected for the study area due to the deficiency of their health infrastructure, high rate of population growth, and high incidence of tropical diseases directly related to the health conditions.

Keywords: indicators; environmental health; sanitation; northern of Brazil.

\section{Introdução}

Segundo Dubos (1965), citado por Heller (1997), "Saúde é o resultado do equilíbrio dinâmico entre o indivíduo e o meio ambiente". Essa definição resume a relação existente entre todos os fatores externos ao indivíduo que exercem pressões sobre o seu bem-estar, levando à ocorrência de agravos à saúde.

A problemática da relação saúde-ambiente é caracterizada pela multidisciplinaridade dos fatores que a compõe. Eles podem ser de ordem política, econômica, social, cultural, psicológica, genética, biológica, física e química. Segundo Rouquayrol (1987), a associação destes fatores é sinérgica ${ }^{1}$, isto é, dois fatores estruturados aumentam o risco de doença mais do que faria a sua simples soma.

Corvalán (2004) define saúde ambiental como sendo os aspectos da saúde humana e das enfermidades que são determinados por fatores ambientais. De acordo com a Funasa (2006), a Organização Mundial da Saúde (OMS) conceitua saúde ambiental como a parte da saúde pública que se ocupa das formas de vida, das substâncias e das

${ }^{1}$ Adjetivo relativo à sinergia. Sinergismo: [Do latim científico synergismus (< gr. synergós, 'que trabalha junto'; 'cooperador', + lat. -ismus)]. Substantivo masculino. Química. Ação cooperativa de duas ou mais substâncias, de modo que o efeito resultante é maior que a soma dos efeitos individuais destas. 
condições em torno do homem que podem exercer alguma influência sobre a saúde e o bem-estar. Desta forma, a conservação e a proteção do meio ambiente assumem papel decisório nas medidas relativas à promoção da saúde nas populações.

A infraestrutura sanitária deficiente desempenha uma nítida interface com a situação de saúde e com as condições de vida das populações dos países em desenvolvimento, nos quais as doenças infecciosas continuam sendo uma importante causa de morbidade e mortalidade (DANIEL, 2001). A prevalência dessas doenças constitui um forte indicativo da fragilidade dos sistemas públicos de saneamento.

Prüss et al (2002) estimaram que a diarreia é responsável por 4,3\% dos anos de vida perdidos ou com incapacitação (DALY) no mundo e que $88 \%$ desta carga de doenças é atribuída ao abastecimento de água, esgotamento sanitário e higiene inadequados. A maior concentração dessa carga recai em crianças dos países em desenvolvimento com condições impróprias de saneamento.

Costa et al (2006) elaborou uma listagem de doenças relacionadas às deficiências de saneamento ambiental, com a finalidade de explorar os Sistemas de Informações de Saúde, conforme a distribuição dessas doenças no Brasil. A Tabela 1 mostra a listagem das doenças.

Segundo Borja e Moraes (2003A), no campo do saneamento ambiental, é urgente a estruturação de um sistema de indicadores para avaliar as condições ambientais, principalmente pela necessidade de se dispor de instrumentos confiáveis que respaldem o planejamento, a execução e a avaliação da ação pública, e não apenas pela fragilidade dos indicadores existentes.

Os indicadores têm como papel principal a transformação de dados em informações relevantes para os tomadores de decisão e o público. Em particular, eles podem ajudar a simplificar um arranjo complexo de informações sobre saúde, meio ambiente e desenvolvimento, possibilitando uma visão "sintetizada" das condições e tendências existentes (VON SCHIRNDING, 2002).

O processo de construção de um sistema de indicadores ambientais envolve uma série de decisões e exige uma concepção integrada do meio ambiente e, consequentemente, uma abordagem interdisciplinar (BORJA; MORAES, 2003B). Essa abordagem deve considerar condições ambientais, demográficas, comportamentais, culturais e socioeconômicas (HELLER; AZEVEDO, 2006).

Tabela 1 - Doenças relacionadas ao saneamento ambiental inadequado

\begin{tabular}{|c|c|}
\hline Categoria & Doenças \\
\hline Doenças de transmissão feco-oral & Diarreia, febre entérica e hepatite $A$ \\
\hline $\begin{array}{l}\text { Doenças transmitidas por } \\
\text { inseto-vetor }\end{array}$ & $\begin{array}{l}\text { Dengue, febre amarela, } \\
\text { leishmaniose Filariose linfática, } \\
\text { malária e doença de chagas }\end{array}$ \\
\hline $\begin{array}{l}\text { Doenças transmitidas } \\
\text { pelo contato com a água }\end{array}$ & Esquistossomose, leptospirose \\
\hline $\begin{array}{l}\text { Doenças relacionadas } \\
\text { com a higiene }\end{array}$ & $\begin{array}{c}\text { Doença dos olhos, tracoma, } \\
\text { conjuntivites, doenças da pele, } \\
\text { micoses superficiais }\end{array}$ \\
\hline Geo-helmintos e teníases & Teníases e helmintíases \\
\hline
\end{tabular}

Fonte: Costa et al (2006). Modificado.
Nesse sentido, este trabalho teve por objetivo a construção de indicadores de saneamento e de saúde ambiental em município do Norte do Brasil. Pretendeu-se compor um sistema com menor quantidade de variáveis para discriminar áreas com distintos níveis sanitário-ambientais, e relacionar os indicadores com a ocorrência de agravos à saúde nessas áreas. Escolheu-se o município de Tucuruí devido à deficiência de sua infraestrutura sanitária, alta taxa de crescimento da população, e ainda, por acreditar-se que este município represente as condições sanitário-ambientais da região Norte do Brasil.

\section{Metodologia}

O município de Tucuruí, apresentado na Figura 1, localiza-se no estado do Pará e possuía, segundo dados do Instituto Brasileiro de Geografia e Estatística (2007), 89.264 habitantes. Segundo Moura (2006), o município apresentava taxa de crescimento populacional de $12 \%$ ao ano, e $98 \%$ da população estava concentrada na área urbana. O produto interno bruto (PIB) é o mais alto do estado devido ao royalty pago pela Usina Hidrelétrica de Tucuruí. A despeito da alta arrecadação do município, a infraestrutura é precária com relação aos serviços de saneamento ambiental.

Duas hipóteses foram formadas a partir do problema de investigação. A primeira diz respeito à possibilidade de se utilizarem indicadores já mencionados na literatura para definição de níveis sanitário-ambientais. A segunda trata da identificação (discriminação) de populações expostas a diferentes níveis sanitário-ambientais e à verificação da relação entre os baixos níveis de saneamento e saúde ambiental levantados e baixos níveis de saúde dos residentes das áreas investigadas.

\section{Delineamento do estudo}

Adotou-se o estudo epidemiológico transversal, realizado em três áreas da cidade de Tucuruí. A escolha das áreas embasou-se na observação e no conhecimento local do município, de acordo com diferentes níveis de infraestrutura sanitária e condições sociais.

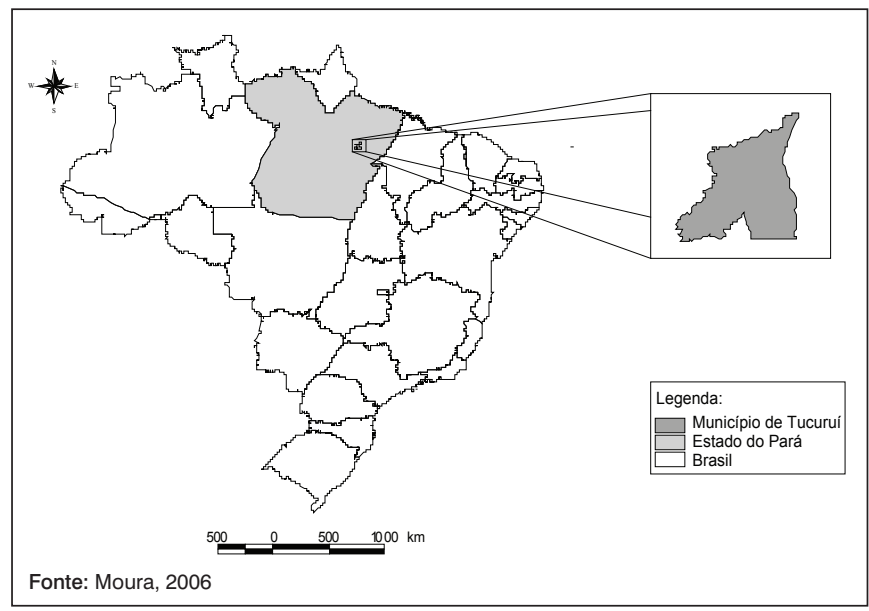

Figura 1 - Localização do município de Tucuruí, PA 
A área A que margeia o rio Tocantins, é considerada o núcleo de desenvolvimento da cidade e está suscetível a inundações. É formada pelos bairros Beira Rio, Castanheira, Colinas, Jaqueira, Jardim Paraíso, Mangal e Matinha.

A área B é composta por bairros que foram formados por invasões de áreas privadas como Alto Alegre, Getat, Jardim Colorado e Terra Prometida. Nessa área a maioria das ruas não é pavimentada.

A área C possui ruas, calçadas, rede coletora de esgotos e drenagem pluvial. É formada pelos bairros Cohab e Jardim das Flores. A área $C$ foi utilizada como comparação entre as demais áreas a fim de se padronizar a comparação entre A e B.

\section{População de estudo e amostragem}

A população de estudo foi calculada assumindo-se a prevalência do fator mais comum no grupo de referência de 50\%, com uma razão de probabilidade em 1,3 e 1,4, na comparação de dois grupos a um erro amostral de $5 \%$ e poder da amostragem de $9 \%$.

A seleção das residências que participaram do estudo foi realizada através de sorteio de 200 domicílios entre a totalidade de residências existentes em cada área. No conjunto das três áreas sorteou-se a totalidade de 600 residências, apresentada na Figura 2. O procedimento de escolha das residências foi o da randomização aleatória simples. Para tanto, utilizou-se o mapa da área urbana de Tucuruí com a localização dos domicílios existentes no ano de 2002, conforme informações fornecidas por imagens de satélite deste ano.

\section{Avaliação dos níveis de saneamento e saúde ambiental}

\section{Entrevistas domiciliares}

Para a coleta de dados, realizou-se um inquérito domiciliar no qual foram aplicados questionários temáticos sobre as condições de saneamento ambiental, condições socioeconômicas, informações sobre o domicílio, condições de saúde ambiental e condições de saúde de cada morador do domicílio.

O questionário foi composto por três partes. A primeira identificava o domicílio e todos os residentes, e continha o termo de consentimento, que era apresentado pelo membro da equipe ao morador entrevistado. Na segunda parte eram levantados dados sobre as condições de saneamento ambiental e socioeconômicas do domicílio. A terceira relacionava-se com as condições de saúde dos residentes.

Levantaram-se informações pertinentes à composição dos indicadores de saúde sobre os óbitos e ocorrência dos agravos (indicadores de mortalidade e morbidade). No caso desse estudo epidemiológico, utilizou-se a prevalência dos agravos na população como indicador de morbidade.

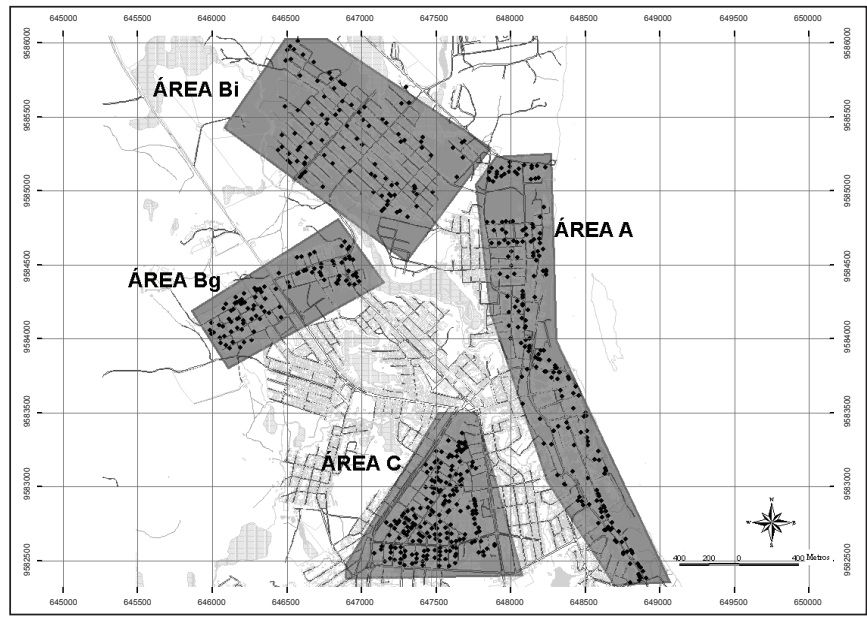

Figura 2 - Áreas e domicílios investigados na área urbana do município

\section{Composição dos indicadores}

Para quantificar os diferentes níveis de saúde e saneamento ambiental, aos quais as populações das diferentes áreas de estudo estavam expostas, gerou-se seis indicadores. Os indicadores foram denominados de escores. Eles representam todas as variáveis relacionadas a um determinado indicador segundo um aspecto sanitário-ambiental que foram passíveis de observação pela equipe de campo na região de estudo. Foi possível, então, relacionar cada indicador com as variáveis que o influenciam e, através de atribuições de pesos específicos para cada um e de análises estatísticas, determinar sua real situação e sua contribuição para o estado da saúde pública local

A Tabela 2 mostra as variáveis da análise dos indicadores de saneamento ambiental. A Tabela 3 mostra as variáveis da análise dos indicadores de saúde.

Para que o agrupamento das variáveis originasse os escores, desenvolveu-se uma rotina, em software de estatística, que atribuía pesos às variáveis de acordo com suas respostas e fez-se o somatório, desses pesos, para cada uma delas. Exemplificando, para a composição do escoreagua, uma das variáveis utilizadas referia-se à existência de água provinda da rede de abastecimento pública; quando a resposta foi "Sim", foi atribuído o valor máximo de peso, dez. Quando a resposta foi "Não" era atribuído o valor mais baixo zero, e assim sucessivamente para todas as variáveis. Para aquelas que possuíam mais alternativas em suas respostas (além de sim e não), a ponderação foi diferenciada, mas dentro do limite mínimo de zero para a pior situação e de dez para a melhor.

Como as unidades de observação do estudo eram os domicílios, cada um deles teve um valor de eescore correspondente ao somatório do peso de cada variável. O valor dos escores de saneamento ambiental, saúde ambiental e pública para cada área investigada foi estimado através da média aritmética dos valores dos escores individuais dos domicílios pertencentes a cada área. 
Tabela 2 - Variáveis da análise com seus respectivos componentes e indicadores de saneamento ambiental

\begin{tabular}{|c|c|c|}
\hline Componentes & Indicadores (Escore) & Variáveis \\
\hline Água de abastecimento & escoreagua & $\begin{array}{l}\text { Domicílios ligados à rede } \\
\text { Falta de água no domicílio } \\
\text { Aplicação de algum tratamento na água antes do consumo } \\
\text { Tipo de tratamento aplicado à água antes do consumo } \\
\text { Local de lavagem das roupas no domicílio } \\
\text { Local de lavagem dos utensílios domésticos no domicílio } \\
\text { Domicílios que possuem chuveiro } \\
\text { Domicílios que utilizam balde para asseio diário } \\
\text { Tipo de abastecimento dos domicílios não ligados à rede } \\
\text { Per capita de água por domicílio }\end{array}$ \\
\hline Esgotamento sanitário & escoresgoto & $\begin{array}{l}\text { Domicílios ligados à rede de esgoto } \\
\text { Domicílios que possuem vaso sanitário } \\
\text { Quantidade de vasos sanitários por domicílio } \\
\text { Domicílios que possuem instalação hidráulica adequada } \\
\text { Disposição do esgoto sanitário no domicílio } \\
\text { Domicílios que utilizam fossa seca }\end{array}$ \\
\hline Resíduos sólidos (lixo) & escorelixo & $\begin{array}{l}\text { Domicílios contemplados com coleta de lixo } \\
\text { Frequência de coleta de lixo no domicílio } \\
\text { Disposição do lixo no domicílio quando não contemplado por coleta } \\
\text { Acomodação do lixo no domicílio } \\
\text { Distância do lixo disposto ao domicílio quando não contemplado com coleta }\end{array}$ \\
\hline Drenagem urbana & escoredren & $\begin{array}{l}\text { Domicílios inundados por água de chuva ou enchente nos últimos cinco anos } \\
\text { Frequência das enchentes } \\
\text { Domicílios com rua pavimentada } \\
\text { Domicílios com rua com sistema de drenagem } \\
\text { Domicílios nos quais a rua sofre alagamento } \\
\text { Alagamento }\end{array}$ \\
\hline
\end{tabular}

Tabelas 3 - Variáveis da análise com seus respectivos componentes e indicadores de saúde

\begin{tabular}{|c|c|c|}
\hline Componentes & Indicadores (Escore) & Variáveis \\
\hline Saúde ambiental & escoreamb & $\begin{array}{l}\text { Domicílios que utilizam ratoeira } \\
\text { Domicílios que utilizam inseticida } \\
\text { Frequência da utilização de inseticida no domicílio } \\
\text { Domicílios que utilizam tela na janela } \\
\text { Domicílios que utilizam mosquiteiro na janela } \\
\text { Domicílios que possuem cão } \\
\text { Domicílios que possuem gato } \\
\text { Quantidade de gatos no domicílio } \\
\text { Quantidade de cães no domicílio } \\
\text { Morte de cães por raiva } \\
\text { Domicílios que possuem porcos } \\
\text { Domicílios que possuem jabuti } \\
\text { Domicílios que tiveram que sacrificar algum animal com leishmaniose } \\
\text { Quantidade de vasos de flores no domicílio } \\
\text { Quantidade de carotes no domicílio } \\
\text { Presença de ratos e camundongos } \\
\text { Presença de moscas no domicílio } \\
\text { Picadas de mosquitos incomodam os moradores do domicílio } \\
\text { Presença do mosquito barbeiro no domicílio } \\
\text { Quantidade de latas no domicílio }\end{array}$ \\
\hline Saúde pública & escorepub & $\begin{array}{l}\text { Indivíduos que já tiveram leptospirose no domicílio } \\
\text { Indivíduos que tiveram doenças da listagem } \\
\text { Indivíduos que tiveram doenças fora da listagem } \\
\text { Óbitos de crianças menores de um ano na família } \\
\text { Óbitos de filhos na família independente da idade } \\
\text { Óbitos de adultos < } 50 \text { anos na família } \\
\text { Óbitos nos últimos cinco anos na família } \\
\text { Todas as variáveis relacionadas à ocorrência de doencas da listagem contidas no questionár }\end{array}$ \\
\hline
\end{tabular}




\section{Análise estatística}

\section{Análise univariada}

Essa análise consistiu na descrição dos domicílios, famílias e residentes das habitações segundo os vários parâmetros estatísticos das variáveis estudadas e na comparação das três áreas segundo as características descritas por essas mesmas variáveis.

Para a descrição dos grupos, construiu-se as distribuições de frequências, calculou-se as médias e desvios padrão e determinouse os percentuais e proporções indicados para cada caso. Quando os critérios de comparação eram variáveis qualitativas, para a comparação das três áreas, os dados foram inseridos em tabelas de contingência tipo RxC. Quando as variáveis utilizadas na comparação eram representadas pelas médias e desvios padrão, foram inseridos os dados em tabelas de ANOVA "oneway". As três áreas foram comparadas conforme a descrição proporcionada por 109 variáveis do estudo. Na comparação das áreas de acordo com as frequências das categorias de variáveis descritas em escala não-numérica, usou-se o teste do $\chi^{2}$ de Pearson para aferir o grau de significância estatística das diferenças observadas.

Na comparação das áreas através das médias das variáveis descritas em escala numérica, utilizou-se o teste de Fischer para tabelas de ANOVA, quando o teste de igualdade de variância de Bartllet esteve associado a $\mathrm{p} \geq 0,05$, e o teste de Kruskall-Wallis quando o teste de igualdade de variância de Bartllet esteve associado a $\mathrm{p} \leq 0,05$.

$\mathrm{Na}$ localização das diferenças observadas entre proporções ou percentagens, quando as comparações baseavam-se em valores apresentados por variáveis não-numéricas, utilizou-se o recurso de remontagem das tabelas de contingência, com a deleção ou reagrupamento das frequências. Nesse caso, o grau de significância estatística foi também aferido por um novo cálculo do $\chi^{2}$.

Foi utilizado o teste $t$ de Student na localização das diferenças observadas entre médias, quando as diferenças simultâneas entre as três áreas foram determinadas pelo teste de Fischer. Para as diferenças simultâneas determinadas pelo teste de Kruskall-Wallis, utilizou-se o estatístico Q, de Dunn para a localização das diferenças mais significativas.

$\mathrm{Na}$ descrição e na comparação das unidades constituídas por dados do domicílio ou família, não se utilizou qualquer medida de ajuste para o desenho amostral. Esses parâmetros foram construídos com dados obtidos em amostragem aleatória simples.

$\mathrm{Na}$ descrição e comparação de entidades formadas com características dos indivíduos, usou-se de algoritmos destinados ao ajuste dos dados às características do modelo amostral utilizados na coleta de dados: cluster ou aglomerado.

As relações possivelmente existentes entre os indicadores de saúde pública e as características de cada uma das áreas foram analisadas pela comparação das probabilidades de se encontrar domicílios com histórias de agravos à saúde entre os seus residentes. Essas relações foram pesquisadas em termos de razões de probabilidade (odds ratio), obtidas quando se determinou as probabilidades de encontrar domicílios com agravos numa área, quando comparados com outra área de referência.

Foram descritas e comparadas as três áreas conforme os valores apresentados pelos domicílios para as seis variáveis que agregavam os efeitos de um número maior de características registradas para essas mesmas unidades. Essas variáveis foram descritas e comparadas, nesse particular, através das médias e desvios padrão obtidos para cada área e escore conforme os padrões de descrição e comparações estatísticas descritas acima. O grau de significância estatística utilizada nessa etapa da análise foi de $5 \%$.

\section{Análise multivariada}

As relações foram estudadas entre os indicadores de saúde pública e outras características das áreas investigadas por meio de modelos de regressão logística. Esses modelos foram construídos com a finalidade de se ajustarem as razões de probabilidade obtidas na etapa univariada da análise à proporção de indivíduos do sexo masculino e feminino existentes no domicílio, a idade média dos residentes, o número médio de pessoas residentes em cada domicílio, e o valor médio dos escores de água de abastecimento, de esgotamento sanitário, drenagem urbana, de resíduos sólidos (lixo) e saúde ambiental. Não foram incluídos na análise os valores médios do escore de saúde pública, uma vez que as variáveis colocadas como termo dependente das equações logísticas estavam também representadas na composição do escore.

$\mathrm{O}$ ajuste das razões de probabilidade verificadas entre cada variável e as três áreas às variáveis incluídas no modelo de regressão foi realizado por algoritmos de entrada de variável passo a passo em cada modelo, tendo-se o nível de significância de 20\% como critério de descarte e manutenção da variável na análise.

O critério utilizado na determinação do grau de significância estatística da associação das variáveis que permaneceram no modelo com a probabilidade do domicílio ser positivo para o agravo de saúde testado foi o de 5\%. A adequabilidade do modelo, medida como capacidade nele incluída em modificar a razão de probabilidade original, foi testada através do $\chi^{2}$ de Hossmer-Lemeshow, admitindo-se como o melhor modelo, aqueles que apresentavam o maior valor de p nesse teste.

\section{Resultados e discussões}

Ao final do trabalho de campo, o número de domicílios e indivíduos incluídos em cada amostra foi de 200 domicílios na área A com 989 indivíduos, 199 domicílios na área B com 997 indivíduos e 199 domicílios na área C com 798 indivíduos.

\section{Características sanitário-ambientais da amostra}

Nas três áreas investigadas encontraram-se, em campo, problemas de infraestrutura urbana como ruas não-pavimentadas, ausência de sistema de drenagem de água pluvial e praticamente nenhuma rede de 
coleta de esgoto. O esgoto e as águas servidas dos domicílios escoavam em valas abertas, pelos próprios moradores, para sua drenagem.

Segundo o Instituto Brasileiro de Geografia e Estatística (2005), 14\% da população de Tucuruí era atendida por rede de esgoto sanitário e a rede de abastecimento de água servia $70 \%$ da população. Dados da Prefeitura revelavam que menos de 30\% do município encontrava-se contemplado com rede de drenagem. Porém, dados coletados in situ mostraram que, da amostra de 598 domicílios, 98\% (586) eram atendidos por rede de abastecimento de água e apenas 2\% (12) dos domicílios eram atendidos por rede de esgoto. A percentagem de domicílios atendidos por coleta de lixo era de $96 \%$.

\section{Águas de abastecimento}

Os domicílios que utilizavam do serviço de abastecimento de água padeciam constantemente com a falta de água. Os moradores restringiam o consumo pela interrupção frequente no abastecimento, e racionavam o uso para desenvolver as atividades domésticas e para seu próprio consumo. Devido a isso, muitas vezes, o asseio diário era feito em igarapés que cortam o município e ou no rio Tocantins, principalmente na área A.

Quanto ao tipo de abastecimento, a área que possuía o maior percentual de domicílios que utilizavam a água da bica para consumo foi a área A, 29,5\%. A área C apresentou o maior número de domicílios que utilizavam água do poço, 106; contra 71 na área B e 65 na área A.

Um recurso utilizado por toda a população do município, e verificado na amostragem, foi a utilização de água da bica, um minadouro de água antigo, que durante muito tempo forneceu água para a população. Segundo a Vigilância Sanitária Municipal, em outubro de 2005 foi detectada a presença de coliformes fecais nessa água.

A maioria da população que consome a água desta bica não faz nenhum tipo de tratamento antes de ingeri-la. Dados da pesquisa revelaram que, dentre os domicílios que afirmaram fazer algum tipo de tratamento antes de consumir a água da bica, a alternativa mais utilizada é simplesmente "coar a água"; com um pedaço de pano. $\mathrm{Na}$ área B, 24\% (48) dos domicílios utilizam esta alternativa, contra 16\% (33) dos domicílios na área A e 9\% (19) dos domicílios na área C.

O uso de poços, nos domicílios, também foi verificado nas áreas investigadas. Encontrou-se a maior concentração na área $C$, na qual 53\% (106) dos domicílios eram providos de poços ou utilizavam água dos poços.

A presença de caixas de água com tampa como forma de armazenamento de água foi comum também em todas as áreas. Esse hábito reflete a preocupação da população com a proliferação da dengue, que é muito frequente no município.

A área B apresentou a maior porcentagem de domicílios ligados à rede de abastecimento de água com 96,5\%. A frequência de falta de água foi classificada como regular em mais de 73\% dos domicílios investigados nas três áreas. Em relação ao percentual de domicílios que faziam algum tipo de tratamento na água antes do consumo, a área B foi a que apresentou o menor percentual com 63,3\%, contra 67\% na área A e 79,4\% na área C.

O tipo de tratamento mais utilizado pelos domicílios foi a filtragem com vela, seguida da coação da água com pedaço de pano. $\mathrm{Na}$ área A, 42,1\% dos domicílios utilizavam filtragem com vela; na área $B$ essa porcentagem era de $35,4 \%$, enquanto que na área $C$ esse valor era de 44,9\%. O uso de coação da água foi mais frequente na área B, com $37,8 \%$ dos domicílios que faziam uso dessa alternativa, contra 24,8\% da área A e 12\% da área C.

\section{Esgoto doméstico}

O esgotamento sanitário era praticamente inexistente, com mais de 97\% dos domicílios nas três áreas afirmando não serem contemplados com esse serviço. Com isso, o uso de alternativas como a fossa seca era muito comum; com a maior concentração dessa alternativa na área B com 32,2\%, contra 21\% na área A e 9,1\% na área C.

A precariedade das instalações sanitárias nos domicílios visitados foi marcante, principalmente nas áreas A e B. Na área B, 32\% (64) dos domicílios visitados não possuíam banheiro com vaso sanitário, contra 21\% (42) de domicílios com banheiro sem vaso sanitário na área A e 9\% (18) dos domicílios com banheiro sem vaso sanitário na área C.

A alternativa utilizada por esses domicílios era o uso de fossas secas, buracos feitos no chão, afastados das edificações.

\section{Resíduos sólidos}

A frequência na coleta dos resíduos sólidos domésticos pode ser considerada alta, com mais de 54\% dos domicílios nas três áreas afirmando serem contemplados com esse serviço três vezes por semana.

A disposição final desses resíduos é o lixão da cidade. Trata-se de uma área aberta, com acesso livre, além de ser desprovida de sistema de drenagem de chorume e de gases. Para acomodação dos resíduos as valas são abertas sem nenhum critério de engenharia. É comum encontrar famílias de catadores residindo nessa área.

\section{Drenagem urbana}

$\mathrm{Na}$ área A, 30\% dos domicílios sofreram com enchentes contra 20,1\% na área B e 9,5\% na área C. A predominância de domicílios que sofreram com inundações na área A já era esperada por ser a área mais baixa do município, estar próxima ao rio Tocantins e possuir histórico de grandes inundações.

\section{Indicadores de saúde e saneamento ambiental}

A Tabela 4 apresenta os resultados obtidos na comparação das três áreas segundo as médias dos escores propostos. Também apresenta 
Tabela 4 - Caracterização das três áreas de estudo, segundo as médias e desvios padrão de escores de saúde pública, saúde e saneamento ambiental obtidos para cada área no inquérito de Tucuruí, e respectivos valores de F/H e $\mathrm{p}$

\begin{tabular}{|c|c|c|c|c|c|c|}
\hline Características da amostra & Atributos & Área $\mathrm{A}$ & Área B & Área C & $\mathrm{F} / \mathrm{H}^{*}$ & $p$ \\
\hline \multirow{2}{*}{ escoreagua } & Média & 49,0 & 45,4 & 56,4 & 27,82 & 0,0000 \\
\hline & $\mathrm{N}$ & 200 & 199 & 199 & & \\
\hline \multirow{2}{*}{ escoresgoto } & Desvio padrão & 15,5 & 17,8 & 11,9 & & \\
\hline & $\mathrm{N}$ & 200 & 199 & 199 & & \\
\hline \multirow[t]{2}{*}{ escorelixo } & Média & 18,8 & 16,8 & 18,6 & 37,62 & 0,0001 \\
\hline & Desvio padrão & 4,8 & 4,1 & 5,0 & & \\
\hline \multirow{2}{*}{ escoredren } & Desvio padrão & 9,22 & 8,5 & 6,2 & & \\
\hline & $\mathrm{N}$ & 200 & 199 & 199 & & \\
\hline \multirow[t]{3}{*}{ escoreamb } & Média & 154,5 & 148,2 & 157,1 & 12,78 & 0,0000 \\
\hline & Desvio padrão & 16,6 & 19,2 & 18,4 & & \\
\hline & $\mathrm{N}$ & 200 & 199 & 199 & & \\
\hline escorepub & Média & 62,8 & 64,8 & 63,6 & 10,72 & 0,0000 \\
\hline
\end{tabular}

*F: teste de Fischer para tabelas de ANOVA e $\mathrm{H}: \chi^{2}$ de Kruskall-Wallis.

os resultados dos testes de significância estatística aplicada a cada comparação e os respectivos valores de p.

$\mathrm{Na}$ localização das diferenças quando se compara as áreas segundo o escore de água de abastecimento (escoreagua), o valor de t (Bonferroni) para a comparação entre as áreas A e B é -3,682 com $\mathrm{p}=0,044$ para as áreas $\mathrm{A}$ e $\mathrm{C}, \mathrm{t}=7,342 \mathrm{e} \mathrm{p}=0,000$ e, para a comparação entre $\mathrm{B}$ e $\mathrm{C}, \mathrm{t}=11,025$ e $\mathrm{p}=0,000$.

A localização das diferenças verificadas entre as áreas para os escores de esgoto, lixo e drenagem foi feita pelo método de Dunn, que é um dos indicados para a localização de diferenças apontadas pelo teste de Kruskall-Wallis quando se compara mais de duas amostras.

Para a localização das diferenças referentes ao escore de esgoto (escoresgoto), o valor de Q (Dunn) para a comparação entre as áreas A e B foi de 1,1898 com p > 0,50; para as áreas A e C o valor de Q (Dunn) foi de 2,874 com $\mathrm{p}<0,02$; e para as áreas B e C o valor de $\mathrm{Q}$ (Dunn) foi de 4,059 com $\mathrm{p}<0,001$.

Quanto ao escore de drenagem (escoredren), o valor de Q (Dunn) para a comparação entre as áreas A e B foi de 3,69 com um $\mathrm{p}<0,001$, para as áreas A e C o valor de Q (Dunn) foi de 3,210 com p < 0,005 e, para as áreas B e C o valor de Q (Dunn) foi de 6,97 com p < 0,001.

Quanto ao escore de lixo, o valor de Q (Dunn) para a comparação entre as áreas A e B foi de 6,01 com um p < 0,001, para as áreas A e C o valor de $Q$ foi de 1,937 com $\mathrm{p}<0,20$ e, para as áreas B e C o valor de $\mathrm{Q}$ foi de 4,07 com $\mathrm{p}<0,001$.

$\mathrm{Na}$ localização das diferenças quanto ao escore de saúde ambiental, o valor de t para a comparação entre as áreas A e B é de -6,414 e $\mathrm{p}=0,001$, entre as áreas $\mathrm{A}$ e $\mathrm{C} t=2,495 \mathrm{com}$ um valor de $\mathrm{p}=0,510$ e entre as áreas B e C, $\mathrm{t}=8,909$ e $\mathrm{p}=0,000$.

Quanto ao escore de saúde pública (escorepub), encontrou-se $t$ (Bonferroni) $=-1,190$ e $\mathrm{p}=1,000$ ao comparar-se as áreas $\mathrm{A}$ e B; $\mathrm{t}=7,146$ e $\mathrm{p}=0$,001 ao comparar-se as áreas A e C; e $\mathrm{t}=8,367$ e $\mathrm{p}=0,000$, ao comparar-se as áreas B e C.
Na comparação das três áreas segundo as médias de escores, apresentado na Tabela 4 verificou-se através do valor de t de Bonferroni que, em relação ao escore de água de abastecimento (escoreagua), as áreas diferiram entre si. Para o escore de esgotamento sanitário (escoresgoto), utilizou-se o valor de Q de Dunn no qual a diferença se concentrou na área C. Quanto ao escore de lixo (escorelixo), também foi utilizado o Q de Dunn com a diferença localizada na área B. Para o escore de drenagem urbana (escoredren), ainda com uso de Q de Dunn, a diferença localizou-se na área C. Para o escore de saúde ambiental (escoreamb), através do valor de t de Bonferroni a diferença se concentrou na área B; para o escore de saúde pública (escorepub), foi utilizado o Q de Dunn, e localizou-se a diferença na área C.

Verificou-se a relação dos níveis de saneamento com as variáveis da Tabela 4 , denominadas de indicadores de saúde e que na comparação das áreas obtiveram valor de $\mathrm{p}<0,05$, ou seja, mostraram-se significativas, indicando a existência de diferenças entre as áreas em relação às suas ocorrências. Porém, a comparação das áreas segundo as médias e os percentuais das variáveis isoladas, não identifica padrões que possam afirmar que a área A é melhor que a área C, ou melhor, que a área B. Observou-se que as condições sanitário-ambientais nas três áreas não são adequadas à promoção da saúde.

\section{Indicadores de saúde pública}

A comparação das médias dos escores apresentadas por domicílios com e sem agravos mostraram que de modo geral, os escores que apresentam melhores níveis de saneamento e saúde ambiental (valores mais altos) estão associados a domicílios que não apresentam história de agravo. E as médias de escores que apresentam piores níveis (valores mais baixos) de saneamento e saúde ambiental estão associadas a domicílios positivos para esse fator. 
Abaixo, estão os indicadores relacionados às doenças de transmissão feco-oral, relacionadas com a higiene e transmitidas por inseto-vetor. O inquérito contemplou uma gama maior de doenças, porém acredita-se que essas seriam mais interessantes para confronto com indicadores de saneamento no município de Tucuruí.

\section{Indicador de morbidade por diarreia}

Mais de 50\% dos domicílios das áreas A e B tiveram histórico positivo em relação à diarreia no período de um ano e, pouco menos de 40\% dos domicílios na área C.

$\mathrm{Na}$ área A há 2,1 vezes mais chances de se encontrar domicílios que tenham indivíduos com esse agravo se comparada à área $\mathrm{C}$. $\mathrm{Na}$ área $\mathrm{B}$, as chances de se encontrar domicílios nessa condição são 1,77 vezes a mais que a área $C$. Esses valores de razão de probabilidade são estatisticamente diferentes.

Baseando-se nessa análise, a área $C$ demonstrou menor probabilidade de ocorrência de domicílios com indivíduos que tiveram diarreia e a área A se mostrou a área com maior probabilidade. A comparação das médias de indivíduos que tiveram diarreia por domicílio confirma esse resultado sendo maior na área $\mathrm{A}$, com 0,28 indivíduos/ domicílio, contra 0,22 na área B e 0,23 na área C.

O exame de possíveis efeitos dos escores de saneamento e saúde ambiental e de outros indicadores importantes nessa análise, como a idade média dos indivíduos por domicílio; sexo dos indivíduos e a quantidade de residentes por domicílio em modelos de regressão logística confirmam essa diferença.

O fato de a área A ter se mantido nessa análise com o valor de sua razão de probabilidade alterada (de 2,1 para 1,77) e continuando significativa, estatisticamente, juntamente com os outros indicadores utilizados na análise, reforça a diferença existente entre ela e as áreas $\mathrm{B}$ e C. Isso mostra que a influência que a área exerce sobre o indicador de saúde é dependente dos outros indicadores, ou seja, a redução da probabilidade de um domicílio na área A possuir indivíduos que tiveram diarreia ocorre com a melhora ou aumento das condições dos domicílios relativas a outros indicadores analisados, nesse caso, os escores de lixo, saúde ambiental e idade média dos indivíduos.

\section{Indicador de morbidade por diarreia em menores de cinco anos}

Sobre o indicador diarreia em menores de cinco anos de idade, a área B exibe um percentual de 21,6\% (43) de seus domicílios positivos em relação a esse indicador, enquanto a área A apresentou o valor de 17\% (34) e a área C, 8\% (16). Na análise não-ajustada, essa comparação foi verificada com o valor da razão de probabilidade para a comparação entre as áreas de 2,3 para a área A e 3,1 para a área B, com esses valores indicando que na área $\mathrm{B}$, as chances de se encontrar domicílios com indivíduos menores de cinco anos que tiveram diarreia foi de 3,1 vezes mais, se comparada a área C. A área A, mostrou uma probabilidade maior da ocorrência desse evento em relação à área $\mathrm{C}$, com 2,3 vezes mais chances, mas com probabilidade menor do que na área B. Essas comparações não-ajustadas possuem significado estatístico, reafirmando a diferença existente entre as áreas, e já indicada pela distribuição de frequência na análise univariada.

$\mathrm{Na}$ análise ajustada, os indicadores que exercem alguma influência na ocorrência desse indicador de saúde são a idade média dos indivíduos da amostra e o escore de saúde ambiental, sendo ambos significativos estatisticamente. As áreas não foram relevantes nessa análise, indicando que a ocorrência do indicador de saúde não é relativa a uma área só, e sim às condições dos domicílios das áreas investigadas em relação às condições da saúde ambiental dos domicílios e à idade dos indivíduos nos mesmos.

Os valores de razão de probabilidade para essas variáveis são 0,82 e 0,98, respectivamente. Esses valores indicam que, com o aumento da idade média dos indivíduos da amostra, há uma redução da ocorrência de domicílios com indivíduos menores de cinco anos que tiveram diarreia, fato esse também observado no valor da razão de probabilidade para o escore de saúde ambiental. Além disso, o menor valor de escore de saúde ambiental foi encontrado na área B quando comparado à área A e C, levando a conclusão de que a área B é a pior referente à ocorrência desse indicador, ou seja, com a maior probabilidade de ocorrência.

O valor do p de Hosmer-Lemeshow para essa análise foi de 0,813, valor esse considerado alto, o que implica na boa adequabilidade do modelo referente à tentativa de explicar a relação analisada.

Gross et al (1989) investigaram o impacto das medidas de saneamento na diarreia em 254 crianças menores de seis anos em duas favelas de Belo Horizonte, e encontraram melhora sensível do percentual de crianças acometidas desse mal após a melhora desses serviços.

\section{Indicador de morbidade por micose}

Sobre o indicador de indivíduos com micose em agosto e setembro de 2005, 30,6\% (61) de domicílios positivos estão localizados na área B, contra 22,5\% (42) na área A e 24,1\% (48) na área C.

$\mathrm{Na}$ análise não-ajustada, o valor da razão de probabilidade indica que a área B é a área na qual há mais chances de se encontrar domicílios com indivíduos que tiveram micose nos últimos dois meses, 1,39 vezes mais chances se comparada à área C. A área A apresenta menor probabilidade com uma razão de probabilidade de 0,91. Essas razões não apresentaram significância estatística, não sendo possível afirmar a existência de diferença, assim como na distribuição de frequência já mencionada anteriormente.

A análise ajustada indica que os indicadores escore de água, escore de drenagem e escore de lixo exercem influência na ocorrência desse indicador de saúde.

Esses indicadores têm uma relação inversamente proporcional com a ocorrência dessa variável, devido aos valores de razão de probabilidade; 0,98, 0,96 e 0,96, respectivamente. Uma melhora das condições desses serviços nos domicílios indica que há redução da probabilidade de se encontrar domicílios com indivíduos que tiveram micose nos últimos dois meses. Desses indicadores, somente o escore de lixo não foi significativo estatisticamente. Dessa forma, os 
outros indicadores podem ser considerados como fatores de risco de ocorrência desse indicador. $\mathrm{O}$ valor de $\mathrm{p}$ de Hosmer-Lemeshow para essa análise foi de 0,999, o que indica que o modelo foi adequado à descrição que se pretendia analisar.

\section{Indicador de morbidade por malária}

Sobre o indicador de saúde referente à ocorrência de malária, um maior percentual de domicílios positivos em relação a esse indicador na área B, com 27\% (55); contra 12\% (24) na área C e 14\% (28) na área $\mathrm{A}$. Os valores de razão de probabilidade não-ajustada para as áreas A e B foram de 1,18 e 2,78, respectivamente.

Esse valor indica que a área $B$ tem 2,78 vezes mais chances de se encontrar domicílios com indivíduos que tiveram malária, no período de um ano, do que na área C. Na área A, essa probabilidade é reduzida para 1,18. Esse valor para a área A é não-significativo estatisticamente, indicando que não se pode afirmar que haja diferença entre ela e a área $\mathrm{C}$, referente à ocorrência desse indicador de saúde.

$\mathrm{Na}$ análise ajustada, o valor da razão de probabilidade indicou uma redução do valor da razão de probabilidade para 1,90 na área B, fato esse ocorrido devido à influência do efeito dos outros indicadores, o escore de saúde ambiental e de lixo; com valores de razão de probabilidade de 0,98 e 0,91, respectivamente.

Esses indicadores indicam que com a melhoria da qualidade desses serviços nos domicílios, há uma redução da probabilidade de encontrar domicílios com indivíduos que tiveram malária no período de um ano.

Como a área B mostrou-se presente na análise ajustada e com valor significativo de sua razão de probabilidade, a existência de diferença entre as áreas é confirmada, com esse valor localizando a diferença na área B.

O número de residentes por domicílios apresentou um valor de razão de probabilidade de 1,14 , o que indica um aumento na probabilidade de ocorrência de indivíduos com malária em famílias mais numerosas. A área B também contém a maior média de residentes por domicílio, com cinco indivíduos/domicílio, contra 4,9 na área A e quatro na área C.

Os indicadores da análise ajustada se mostraram significativos estatisticamente podendo ser considerados como possíveis fatores de risco em relação à ocorrência desse indicador de saúde.

Com isso, a área B se mostra como aquela com maior probabilidade de encontrar domicílios com indivíduos que tiveram malária no período de um ano. Essa análise apresentou valor de p para o teste de Hosmer-Lemeshow no valor de 0,918, com alta adequabilidade do modelo de regressão na descrição da relação investigada.

O sexo também é um fator relevante na ocorrência desse indicador de saúde, no qual há uma relação diretamente proporcional referente ao sexo feminino, com o aumento de casos de indivíduos com pediculose referente a esse sexo por domicílio. O valor da razão de probabilidade para o indicador de indivíduos do sexo feminino por domicílio foi de 5,01.

Os indicadores da análise ajustada se mostraram significativos, podendo ser considerados possíveis fatores de risco à ocorrência desse indicador.
$\mathrm{O}$ valor de $\mathrm{p}$ de Hosmer-Lemeshow foi de 0,538, indicando uma adequabilidade média do modelo na descrição da relação investigada.

\section{Indicador de morbidade por dengue}

Com relação ao indicador de saúde referente a indivíduos com dengue, as áreas A e C apresentaram um pouco mais de 50\% de domicílios positivos para esse indicador, enquanto a área B teve o percentual um pouco acima de $45 \%$.

$\mathrm{Na}$ análise não-ajustada, o valor da razão de probabilidade indicou que a área $\mathrm{A}$, se comparada à área $\mathrm{C}$, tem 1,01 vezes mais chances de se encontrar domicílios com indivíduos com dengue, se comparada à área C. A área B já apresentou uma redução dessa probabilidade, com o valor de razão de probabilidade de 0,83. Essas razões de probabilidade mostraram-se sem significância estatística, não podendo afirmar que há diferença entre as áreas em relação à ocorrência desse indicador.

O valor da razão de probabilidade ajustada, para o escore de lixo e saúde ambiental, 0,92 e 0,98 respectivamente, indicam que quanto melhor as condições do domicílio nesses aspectos, menor a probabilidade de se encontrar domicílios com indivíduos que tiveram dengue. Penna (2003) ressaltou a relevância da atuação conjunta da área de saneamento e meio ambiente na prevenção da dengue. A redução do valor da razão de probabilidade na área B revela a influência do efeito dos outros indicadores na análise.

O último indicador que tem alguma relação com esse é o sexo dos indivíduos, que, em relação ao indicador de indivíduos do sexo feminino utilizado na análise, apresentou um valor de razão de probabilidade de 0,41, o que indica uma probabilidade maior de se encontrar domicílios com indivíduos que tiveram dengue do sexo masculino.

Todos os indicadores na análise ajustada são significativos estatisticamente, podendo ser considerados como possíveis fatores de risco à ocorrência desse indicador.

Essa análise apresentou valor de p de Hosmer-Lemeshow de 0,162 , indicando que a adequabilidade do modelo de regressão foi baixa, considerado portanto um modelo não adequado para a descrição dessa relação. Apesar disso, esse indicador foi um dos que se mostraram significativos na média com a maior ocorrência de indivíduos que tiveram esse agravo por domicílio na área A.

\section{Indicador de morbidade por febre amarela}

$\mathrm{O}$ indicador de febre amarela apresentou uma percentagem de 0,50\% (1) de domicílios positivos para as áreas A e C, e para a área B, o valor foi de $1 \%$ (2).

$\mathrm{Na}$ análise não-ajustada, o valor da razão de probabilidade para a área B, indica que há 2,01 vezes mais chances de se encontrar indivíduos com febre amarela na área $\mathrm{B}$, se comparada à área $\mathrm{C}$. Na área $\mathrm{A}$, o valor da razão de probabilidade foi de 0,99, indicando probabilidade menor da ocorrência dessa variável nessa área se comparada à área C. Como essas razões de probabilidade não se mostraram significativas estatisticamente, não é possível afirmar que há diferença entre as áreas. 
O escore de drenagem, na análise ajustada, foi o único indicador que tem alguma influência na ocorrência do indicador de saúde. O valor da razão de probabilidade para o escore é de 0,90, o que significa que com a melhoria desse serviço no domicílio há uma redução da probabilidade da ocorrência de domicílios com indivíduos que tiveram febre amarela. Essa é uma informação relevante, já que a febre amarela é considerada uma doença bem controlada no país. Este indicador se mostrou significativo estatisticamente, podendo ser considerado com um possível fator de risco à ocorrência desse agravo.

O valor de p para essa análise foi de 0,414, indicando alguma adequabilidade do modelo na descrição da relação investigada, mas não descrevendo-a ainda da melhor forma.

\section{Conclusões}

Na relação dos indicadores de saneamento e saúde ambiental com os indicadores de saúde, foi possível identificar os fatores de risco que poderiam influenciar na ocorrência dos agravos por meio da determinação da razão de probabilidade (ou odds ratio) para os indicadores resultantes da análise ajustada.

$\mathrm{A}$ área $\mathrm{B}$ apresentou os piores resultados relacionados à ocorrência dos indicadores de saúde, a área $C$ apresentou os melhores resultados e a área A oscilou entre os piores e os melhores resultados, em alguns indicadores.

A concentração das piores condições sanitário-ambientais na área B não significa que as condições das outras áreas sejam melhores. Esta área, por ter sido invadida, não possui qualquer infraestrutura para habitação. A infraestrutura sanitário-ambiental é igualmente deficiente em todo o município.

\section{Agradecimentos}

Os autores agradecem ao Conselho Nacional de Desenvolvimento Científico e Tecnológico (CNPq) pela concessão de auxílio financeiro, (processo 472283/2004-9).

\section{Referências}

BORJA, P.C.; MORAES, L.R.S. Indicadores de saúde ambiental com enfoque para a área de saneamento. Parte 1 - aspectos conceituais e metodológicos. Revista Engenharia Sanitária e Ambiental, Rio de Janeiro, v. 8, n. 1, p. 13-25, jan./mar., 2003A.

Indicadores de saúde ambiental com enfoque para a área de saneamento. Parte 2 - estudo de caso. Revista Engenharia Sanitária e Ambiental, Rio de Janeiro, v. 8, n. 2, p. 26-38, abr./jun., 2003B.

CORVALÁN, C. Processos de toma de decisiones en Salud Ambientale. Brasília: World Health Organization, 2004.

COSTA, M.A. et al. Impactos na Saúde e no Sistema Único de Saúde decorrentes de agravos relacionados a um saneamento ambiental inadequado. In: Fundação Nacional da Saúde. $1^{\circ}$ Caderno de pesquisa em engenharia de saúde pública. 2. ed. Brasília: Funasa, 2006. p. 7-27.

DANIEL, L.A. (Coord.). Processos de desinfecção e desinfetantes alternativos na produção de água potável. Projeto Prosab, Rio de Janeiro: Rima/ABES, 2001.

DUBOS, R. Man adapting. New Haven: Yale Press University, 1965.

FUNASA. Programa de cooperação técnica. Fundação Nacional de Saúde, Brasília: Funasa, 2006.

GROSS, R.; SCHELL, B.; MOLINA, M.C.B. O impacto das medidas de melhoria do abastecimento de água e esgoto sobre a diarréia e parasitose intestinal: experiência brasileira com crianças de comunidades de baixa renda. Revista de Saúde Pública, v. 23, n. 3, p. 214-220, jun. 1989.
HELLER, L. Saneamento e saúde. Organização Panamericana de Saúde da Organização Mundial da Saúde. Representação do Brasil. Brasília, 1997.

HELLER, L.; AZEVEDO, E.A. Exclusão sanitária em Belo Horizonte, MG: caracterização e associação com indicadores de saúde. In: Fundação Nacional da Saúde. $1^{\circ}$ Caderno de pesquisa em engenharia de saúde pública. 2. ed. Brasília: Funasa, 2006. p. 71-98.

INSTITUTO BRASILEIRO DE GEOGRAFIA E ESTATÍSTICA. Censo 2000 Disponível em: < http://www.ibge.gov.br/cidadesat/topwindow.htm?1>. Acesso em jul. 2007.

Atlas de saneamento. Disponível em: <http://www.ibge.gov.br/ home/estatistica/ populacao/atlas saneamento/>. Acesso em 20 de mar. de 2005 .

MOURA, R.B.S. Análise sanitário-ambiental da exposição da população em Tucuruí, PA. Dissertação (Mestrado) - Escola de Engenharia de São Carlos da USP, São Carlos, 2006.

PENNA, M.L.F. Um desafio para a saúde pública brasileira: o controle do dengue. Cadernos de Saúde Pública, v. 19, n. 1, p. 305-309, jan./fev. 2003.

PRÜSS, A. et al. Estimating the burden of disease from water, sanitation, and hygiene at a global level. Environmental Health Perspectives, v. 110 n. 5, p. 537-542, 2002.

ROUQUAYROL, M.Z.; ALMEIDA FILHO, N. Epidemiologia e saúde. 2. ed. Rio de Janeiro: Medsi, 1987

VON SCHIRNDING. Health in sustainable development planning: the role of indicators. Geneva: World Health Organization, 2002. 